\title{
Annual Meeting Abstracts of the German Society of Skeletal Radiology (DGMSR) 2021, April 23 - 24, Berlin/ Germany
}

\author{
Scientific Session Program
}

Oral presentations

Compositional MRI Beyond Structure and Inflammation Ankylosing Spondylitis Is Associated with Proteoglycan Loss of the Lumbar Intervertebral Disks

Daniel B. Abrar, Christoph Schleich, Styliani Tsiami, Miriam Frenken, Gerald Antoch, Philipp Sewerin, Juergen Braun, Sven Nebelung, Xenofon Baraliakos. Dusseldorf, Germany; Herne, Germany

Purpose: To compare the glycosaminoglycan (GAG) content of intervertebral discs (IVDs) of lumbar spines of patients with ankylosing spondylitis (AS) and healthy volunteers, and to assess the relationship between GAG loss and disease-related features.

Materials and Methods: 50 AS patients (mean age $50 \pm 10.5$ years) and 30 age-matched volunteers underwent 3-T magnetic resonance imaging (MRI) and conventional radiography (CR). The MRI protocol included high-resolution morphological and GAG-Chemical-ExchangeSaturation-Transfer (gagCEST) sequences of lumbar spines. Morphological images were evaluated for disk degeneration, inflammation, fatty deposits, and structural changes. Clinical and serological markers included and Bath AS Function (BASFI) and Bath AS Disease Activity (BASDAI) indices, and C-reactive protein (CRP) levels. GagCEST values were compared using a linear mixed model.

Results: GagCEST values were significantly lower in patients with AS $(2.0 \pm 1.7 \%)$ than in healthy subjects $(2.4 \pm 1.8 \%), \mathrm{p}=0.001$. Small, but significant correlations were found between gagCEST values and CRP values $(\tau=-0.14, p=0.007)$, BASFI $(\tau=-0.18, p<0.001)$ and the presence of syndesmophytes $(\tau=-0.17, p=0.001)$. No significant correlations were found with inflammation, fat deposition MRI scores, and BASDAI.

Conclusion: Lumbar IVDs of patients with AS suffer from significant GAG depletion, which is associated with syndesmophyte formation, functional disability and elevated serum CRP-levels. In addition to elucidating the pathophysiological role of cartilage in AS, these results suggest that gagCEST imaging could play an additional confirmatory role in the assessment of disease-related pathological MRI findings in AS.

Diagnosis of joint invasion in patients with malignant bone tumors: value and reproducibility of direct and indirect signs on MR imaging

Jannis Bodden, Jan Neumann, Alexander A. Fingerle, Michael Rasper, Carolin Knebel, Rüdiger v. Eisenhart-Rothe, Carolin Mogler, Katja Specht, Christine Bollwein, Benedikt J. Schwaiger, Alexandra S. Gersing, Klaus Wörtler. Munich, Germany

Purpose: To evaluate the performance and reproducibility of MR imaging features in the diagnosis of joint invasion (JI) by malignant bone tumors.
Materials and Methods: Pretherapeutic MR examinations of patients with $(n=24)$ and without $(n=24)$ JI were assessed by three independent readers, blinded for pathologic outcomes. On MR images, direct (intrasynovial tumor tissue (ITT), intraarticular destruction of cartilage/ bone, invasion of capsular/ligamentous insertions) and indirect signs (tumor size, signal alterations of epiphyseal/transarticular bone (bone marrow replacement/edema-like), synovial contrast enhancement, joint effusion) of JI were assessed. Diagnostic performance of imaging features was analyzed using exact logistic regression models. Sensitivities, specificities, positive (PPV) and negative predictive values (NPV) were calculated, and inter- and intra-reader reproducibility was determined using Fleiss' and Cohen's $\mathrm{K}$.

Results: Tumors were located at the shoulder $(n=7)$, hip $(n=14)$ or knee $(\mathrm{n}=27)$. Readers assessed the presence of JI with high sensitivity (92\%$100 \%)$ and specificity $(88 \%-100 \%)$. Direct visualization of ITT was the best predictor for JI $(\mathrm{OR} \geq 21.25, \mathrm{P}<0.01$, for each reader) and was also highly accurate and reproducible (sensitivity, specificity, PPV, $\mathrm{NPV}=92 \%-100 \% ; \mathrm{k}=0.95$ ). Synovial contrast enhancement and epiphyseal marrow replacement were sensitive indirect signs (87\%-96\%), however, both lacked specificity (29\%-54\%). Combining highly specific direct signs further increased sensitivity $(96 \%)$ while preserving high specificity (100\%).

Conclusion: Joint invasion by malignant bone tumors can accurately be assessed on preoperative MR images with excellent sensitivity, specificity and reproducibility. Direct visualization of ITT is the most valuable direct sign, while indirect signs are less accurate. Combining multiple specific signs further increases the sensitivity while maintaining high specificity.

MRI for exclusion of ligamentous injury in patients with lateral atlantodental interval asymmetry on initial trauma CT - a diagnostic benefit?

Christoph H. Endler, Daniel Ginzburg, Alexander Isaak, Anton Faron, Patrick A. Kupczyk, Ulrike I. Attenberger, Julian A. Luetkens. Bonn, Germany

Purpose: Cervical spine (CS) CT is regularly performed to exclude cervical spine injury (CSI) within initial evaluation of trauma patients. Thereby, patients with asymmetry of the lateral atlantodental interval (LADI) often receive subsequent MRI to rule out ligamentous injury. This study aimed to evaluate the diagnostic benefit of additional MRI in trauma patients with LADI asymmetry and lack of proven CSI.

Materials and Methods: Patients who received CS CT within initial trauma evaluation between 03/2017-08/2019 were retrospectively evaluated. Among those, patients who underwent subsequent MRI due to LADI asymmetry $\geq 1 \mathrm{~mm}$ with no other signs of CSI were identified and re-evaluated by two blinded readers regarding possible ligamentous injuries.

Results: Among 1553 patients evaluated, 146 (9.4\%) presented with LADI asymmetry $\geq 1 \mathrm{~mm}$. Thereof, 46 patients $(39 \pm 22 \mathrm{y}, 28$ men; mean 
LADI asymmetry $2.54 \pm 1.33 \mathrm{~mm}$ ) underwent supplementary MRI with no other signs of CSI on initial CT. 10/46 patients showed cervical tenderness on clinical examination, while $34 / 46$ patients were asymptomatic. In 2/46 patients, MRI revealed ligamentous injury; both patients showed LADI asymmetry $>3 \mathrm{~mm}$ together with cervical tenderness and received treatment for ligamentous injury. In 13/46 patients, signal alterations of alar ligaments without signs of rupture were observed. Of those, 4 patients were subsequently treated as for ligamentous injury, although they were asymptomatic.

Conclusion: Subsequent MRI following trauma CT of the cervical spine in patients with LADI asymmetry may have diagnostic benefit in symptomatic patients only. In asymptomatic patients without proven CSI, MRI did not reveal any diagnostic benefit and even may lead to overtreatment.

\section{MR Imaging Detection of Adhesive Capsulitis of the Shoulder Essentially Improves from Intravenous Contrast Administration}

Bernd Erber, Andrea Baur-Melnyk, Christian Glaser, Sophia Goller, Georg Öttl, Andreas Heuck. Munich, Germany

Purpose: Adhesive capsulitis (AC) may be easily overlooked in routine shoulder MRI. However, correct identification has important impact on proper therapy. Aim of our study was to investigate the potential advantage of i.v. contrast administration for MRI diagnosis of AC.

Materials and Methods: 150 patients were included in a retrospective study: 50 subjects with at least 3 of 4 clinical signs of AC (reduced abduction and external rotation, positive Apley scratch test, shoulder pain); and 100 patients with other shoulder disease but no history or clinical signs of AC. All patients had MRI with $3 \mathrm{~mm}$ thick T1w sagittal, IW coronal and axial and T1w fatsat sagittal sequences after i.v. contrast. MRIs were independently evaluated for presence/absence of AC by two radiologists with different experience and blinded to clinical and individual information; also, levels of diagnostic confidence were assessed (5point-scale). First, non-enhanced images were read alone and 3 weeks later together with CE images. Data analysis included McNemar's test and U-test $(\mathrm{p}<0.05)$.

Results: Using non-enhanced MRI, readers 1 and 2 correctly diagnosed AC with a sensitivity of 60,4 and $72,9 \%$ and a specificity of 75,5 and $93,9 \%$, respectively. Specificity was significantly higher in the experienced reader. By additional use of $\mathrm{CE}$ sequences sensitivity significantly increased to $81,2 \%$ in reader 1 and $87,5 \%$ in reader $2(\mathrm{p}<0,05)$, and specificity to $93,9(\mathrm{p}<0,05)$ and $96,9 \%$ (n.s.), respectively. Individual diagnostic confidence in the diagnosis of $\mathrm{AC}$ improved significantly with CE imaging in both readers $(\mathrm{P}<0,05)$.

Conclusion: Contrast enhanced sequences can significantly increase MRI's sensitivity and specificity as well as radiologist's confidence in the diagnosis of AC. In addition, reader's experience seems to be relevant.

\section{Soft tissue masses: Distribution of entities and rate of malignancy in small lesions}

Felix G. Gassert, Florian T. Gassert, Katja Specht, Carolin Knebel, Ulrich Lenze, Marcus R. Makowski, Rüdiger von Eisenhart-Rothe, Alexandra S. Gersing, Klaus Woertler. Munich, Germany

Purpose: To retrospectively investigate the distribution of histopathologic diagnosis to be encountered in small soft tissue tumors $(\leq 5 \mathrm{~cm})$ in a large series of a tertiary referral center.

Materials and Methods: Patients with a soft tissue mass (STM) with a maximum diameter of $5 \mathrm{~cm}$ presenting at our institution over a period of 10 years, who had undergone preoperative Magnetic resonance imaging and consequent biopsy or/and surgical resection, were included in this study. A final histopathological diagnosis was available in all cases. The maximum tumor diameter was determined on MR images by one radiologist. Moreover, tumor localization (head/neck, trunk, upper extremity, lower extremity, hand, foot) and depth (superficial / deep to fascia) were assessed.
Results: In total, histopathologic results and MR images of 1753 patients were reviewed. 870 patients $(49.63 \%)$ showed a STM $\leq 5 \mathrm{~cm}$ and were therefore included in this study (46.79+/- 18.08 years, 464 women). Mean maximum diameter of the assessed STMs was $2.88 \mathrm{~cm}$. Of 870 analyzed lesions $\leq 5 \mathrm{~cm}, 170(19.54 \%)$ were classified as superficial and $700(80.46 \%)$ as deep. The malignancy rate of all lesions $\leq 5 \mathrm{~cm}$ was at $22.41 \%$ (superficial: $23.53 \% /$ deep: $22.14 \%$ ). The malignancy rate dropped to $16.49 \%(20.79 \% / 15.32 \%)$ when assessing lesions $\leq$ $3 \mathrm{~cm}(\mathrm{p}=0.007)$ and to $15.0 \%(18.18 \% / 13.79 \%)$ when assessing lesions $\leq 2 \mathrm{~cm}(\mathrm{p}=0.006)$. Overall, lipoma was the most common benign lesion of superficial STMs $(29.41 \%)$ and tenosynovial giant cell tumor was the most common benign lesion of deep STMs (23.29 \%). Undifferentiated pleomorphic sarcoma was the most common malignant diagnosis among both, superficial $(6.47 \%)$ and deep (4.29 \%) STMs.

Conclusion: The rate of malignancy decreased significantly with tumor size in both, superficial and deep STMs. The distribution of entities was different between superficial and deep STMs, yet there was no significant difference found in the malignancy rate.

MR-based Proton-Density Fat Fraction (PDFF) differentiates between patients with and without osteoporotic vertebral fractures and between osteoporotic and traumatic vertebral fractures

Florian T. Gassert, Alexander Kufner, Felix G. Gassert, Yannik Leonhardt, Sophia Kronthaler, Benedikt J. Schwaiger, Christof Böhm, Marcus R. Makowski, Jan S. Kirschke, Thomas Baum, Dimitrios C. Karampinos, Alexandra S. Gersing. Munich, Germany

Purpose: To evaluate whether magnetic resonance (MR) imaging-based bone marrow Proton-Density Fat Fraction (PDFF) can differentiate between patients with and without acute osteoporotic/osteopenic vertebral fractures and whether PDFF values can differentiate between low-energy osteoporotic/osteopenic and high-energy traumatic vertebral fractures.

Materials and Methods: Of 52 study participants, 25 presented with one or multiple acute low-energy osteoporotic/osteopenic vertebral fractures of the lumbar spine $\left(\mathrm{BMD}<120 \mathrm{~g} / \mathrm{cm}^{3}\right)$ and 7 patients with acute high-energy traumatic vertebral fractures $\left(B M D>120 \mathrm{~g} / \mathrm{cm}^{3}\right)$. These patients were frequency-matched for age and sex to subjects without vertebral fractures $(N=20)$. Bone mineral density (BMD) values were derived from quantitative CT. Chemical shift encoding-based water-fat MR imaging of the lumbar spine was performed considering a single T2* decay and PDFF maps were calculated. Segmentations of the vertebral bodies were performed excluding fractured vertebrae. Associations between category/fracture status and PDFF were assessed using multivariable linear regression models. Results: A significant correlation between mean PDFF and BMD ( $\mathrm{r}=-$ $0.664, P<0.001)$ was found. In the osteoporotic/osteopenic group, those patients with osteoporotic/osteopenic fractures had a significantly higher PDFF than those without osteoporotic fractures after adjusting for age, sex and BMD (adjusted mean difference [95\% confidence interval]: $19.87 \%[13.23 \%, 26,50 \%] ; P<0.001)$. When evaluating all patients with acute vertebral fractures, those with high-energy traumatic fractures had a significantly lower mean PDFF than those with low-energy osteoporotic/ osteopenic vertebral fractures $(P<0.001)$.

Conclusion: MR-based PDFF enables the differentiation between patients with and without osteoporotic/osteopenic vertebral fractures as well as between low-energy osteoporotic/osteopenic and high-energy traumatic vertebral fractures, suggesting its potential as a biomarker for bone fragility.

MR imaging after patellar MACI and MPFL reconstruction: Comparing isolated with combined procedures

Paula Giesler, Christian Holwein, Julia Schneider, Matthias Jung, Dimitrios C. Karampinos, Dominik Weidlich, Alexandra S. Gersing, Frederic A. Baumann, Andreas B. Imhoff, Fabian Bamberg, Pia M. Jungmann. Freiburg, Germany; Munich, Germany 
Purpose: To qualitatively and quantitatively evaluate whether the 2-year MRI outcome after isolated MPFL reconstruction for treatment of patella instability and after isolated matrix-associated autologous chondrocyte implantation (MACI) for treatment of focal cartilage defects at the patella is better than after combined procedures.

Materials and Methods: In 66 patients with MACI at the patella (cartilage repair, $C R ; n=16$ ), MPFL reconstruction (MPFL; $n=31$ ) or combined surgery (CR\&MPFL; $n=19)$ MRI was performed 2 years after surgery. Clinical scores (KOOS, Lysholm), morphological MRI scores and postoperative cartilage T2 and T1rho relaxation time measurements were obtained.

Results: Overall MRI outcome was good (WORMS progression: $2.3 \pm 6.5$; MOCART: $68.4 \pm 29.5$ ). WORMS at follow up was significantly worse in the CR\&MPFL group $(8.7 \pm 4.9)$ than in the

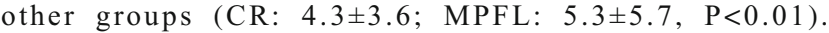
Particularly, patellar cartilage defects were more severe in the CR\&MPFL group $(\mathrm{P}<0.01)$. MOCART scores were significantly better in the CR group than in the CR\&MPFL group ( $87.8 \pm 8.9$ vs $56.6 \pm 27.5, \mathrm{P}<0.001)$. Perfect defect filling was achieved in $69 \%$ and $26 \%$, respectively $(\mathrm{P}=0.031)$. T2 values were higher in both, the CR\&MPFL group (T2 global: $34.0 \pm 2.8 \mathrm{~ms}$; T2 patella: 36.7 $\pm 5.2 \mathrm{~ms})$ and in the CR group $(35.5 \pm 3.1 \mathrm{~ms} ; 35.7 \pm 2.4 \mathrm{~ms})$ as compared to the MPFL group $(31.1 \pm 3.2 \mathrm{~ms} ; 33.5 \pm 3.1 ; \mathrm{P}<0.05)$. Lower T2 values correlated significantly with better clinical scores $(\mathrm{P}<0.05)$.

Conclusion: In case of combined surgery, inferior CR tissue quality and more severe cartilage matrix degeneration and degenerative changes at the knee occur. Therefore, patients with need for combined surgery may be at particular risk for early osteoarthritis.

\section{Intravoxel incoherent motion (IVIM) MR perfusion imaging of de- layed onset muscle soreness (DOMS) and assessment of the effect of compression garments on muscle perfusion}

Rafael Heiss, Frederik Bernd Laun, Svenja Alina Höger, Michael Uder, Bernhard Hensel, Raimund Forst, Thilo Hotfiel, Frank W. Roemer, Andreas Riexinger. Erlangen, Germany; Osnabrück, Germany

Purpose: Aim was to evaluate muscle perfusion in delayed onset muscle soreness (DOMS) by MR perfusion imaging using intravoxel incoherent motion (IVIM) and to assess the effect of compression garments on muscle perfusion.

Materials and Methods: MRI of both lower legs of 16 volunteers was performed before and after DOMS-inducing eccentric exercises. A compression garment was worn during and for $6 \mathrm{~h}$ after exercise on one randomized leg. IVIM MR imaging represented as total muscle perfusion $\mathrm{D} * \mathrm{f}$, perfusion fraction $\mathrm{f}$ and tissue diffusivity $\mathrm{D}$ were compared between baseline, directly, $30 \mathrm{~min}, 6 \mathrm{~h}$ and $48 \mathrm{~h}$ after exhaustion exercise with and without compression. $\mathrm{T} 2 \mathrm{w}$ fs images were acquired at baseline and after 48 hours.

Results: DOMS was induced in the medial head of the gastrocnemius (MGM) muscle in all volunteers. Compression had no effect on IVIM parameters at any time in the MGM or the tibialis anterior muscle. Microvascular perfusion in the MGM increased significantly in both, the compressed and non-compressed leg directly after and 30min after the exercise. Normalization to baseline values occured at 6 hours and was sustained after 48 hours.

Conclusion: Compression garments do not alter muscle perfusion at rest nor have any significant effect during regeneration of DOMS. In DOMS, increased muscle perfusion is observed up to $30 \mathrm{~min}$ after exercise with normalization occuring after $6-48 \mathrm{~h}$. The normalization of perfusion after such a short interval independently of compression may have potential implications regarding the understanding of pathophysiological pathways in DOMS, which may eventually result in tailored therapeutic approaches minimizing the clinical impact of DOMS.
Superior and secondary cleft signs in athletic groin pain - correlation of dedicated MRI and symphysography findings as well as anterior pelvic ring instability

Norman Holl, Judith Sarah Gerhardt, Andres Arevalo-Hernandez, Thomas Tischer, Robert Lenz, Jens Krüger, Marc-André Weber. Rostock, Germany; Berlin, Germany

Purpose: The objective of this ongoing study was to correlate injury patterns such as superior and secondary cleft signs of dedicated MRI with targeted fluoroscopy guided contrast agent injection into the symphyseal cleft in patients with athletic chronic groin pain.

Materials and Methods: Until present 40 sport-active men (mean age 33.5 years; 34 soccer players) were included. Other reasons for groin pain were excluded by a senior surgeon. Standing single leg stance radiography and dedicated 3 Tesla MRI protocol was performed. Symphyseal cleft injection was performed with fluoroscopic guidance. The presence of the superior cleft sign, secondary cleft sign, and osteitis pubis as defined by Byrne et al. (AJR 2017; 209:380-388) was recorded.

Results: Mean symptom duration was 11,5 months and parasymphyseal bone marrow edema was present in 32 of 40 patients (bilaterally in 22 patients). Superior cleft sign was seen in 18 and secondary cleft sign in 20 patients. In 8 patients both cleft signs were present simultaneously.

In 6 cases the cleft was seen in MRI but not in symphysography ( 5 superior cleft, 1 secondary cleft). Anterior pelvic ring instability was observed in 15 patients and was linked with a superior cleft sign in 7 and secondary cleft sign in 6 patients.

Conclusion: Dedicated 3 Tesla MRI outmatches symphysography for purely diagnostic purposes of superior and secondary clefts. Additional symphysography may be important for therapeutic injection of corticosteroids and local anaesthetics. Microtearing at the prepubic aponeurotic complex is a prerequisite of the development of anterior pelvic ring instability.

Structured reporting of CT scans of patients with trauma leads to faster, more detailed diagnoses

Tobias Jorg, Julia C. Heckmann, Philipp Mildenberger, Felix Hahn, Christoph Düber, Peter Mildenberger, Roman Kloeckner, Florian Jungmann. Mainz, Germany

Purpose: To evaluate whether structured reports (SRs) had a positive influence on reporting time or level of detail in trauma CT scans, compared to free-text reports (FTRs).

Materials and Methods: In an experimental study, eight radiology residents used SRs and FTRs to describe 14 whole-body CT scans of patients with polytrauma. Each radiologist created a brief report and a detailed report for each case, with either the SR or an FTR in a simulated emergency room setting. We measured the time to complete the detailed reports of SRs and FTRs. A scoring system was established to perform objective measurements of report completeness and level of detail. Scoring sheets divided the CT findings into main and secondary criteria findings.

Afterwards, the radiologists completed a questionnaire regarding their opinions on the SRs and FTRs.

Results: Radiologists completed the detailed SRs significantly faster than the detailed FTRs (mean 19 min vs. 25 min; p<0.001). The maximum 25min time allowance was used for $25 \%$ of SRs and for $59 \%$ of FTRs. In brief reports, the SRs contained more secondary criteria findings than the FTRs $(\mathrm{p}=0.001)$. No significant differences could be detected in main criteria findings.

Radiologists rated their own SRs as more time-efficient, more concise, and more clearly structured compared to FTRs. Quality, accuracy, and completeness were rated similarly for SRs and FTRs.

Conclusion: We found that SRs for whole-body trauma CTs could add clinical value, compared to FTRs, since use of SRs reduced reporting time and increased the level of detail in trauma CT scans. 
Posterior Extraarticular -ischiofemoral Femoroacetabular Impingement in patients with increased femoral version: using dynamic impingement simulation as a diagnostic tool

Till Lerch, Florian Schmaranzer, Simon Steppacher, Klaus Siebenrock, Moritz Tannast. Bern, Switzerland

Purpose: Posterior extraarticular femoroacetabular impingement (FAI) is poorly understood. These patients present clinically with a lack of external rotation and extension. We asked if range of motion (ROM), location of posterior intra-and extraarticular FAI and the prevalence of posterior extraarticular FAI differs between three groups using patient-specific 3D models based on 3D-CT.

Materials and Methods: Osseous patient-specific 3D models based on 3D CT scans were reconstructed of 52 hips (38 patients) with a positive posterior impingement test and increased $\mathrm{FV}>35^{\circ}$. These included 21 hips with increased $\mathrm{FV}>35^{\circ}$ and 22 hips with increased $\mathrm{FV}>35^{\circ}$ and increased acetabular version $(\mathrm{AV})>25^{\circ}$.

Mean age of the patients was 23 - 38 years. Surgical treatment was performed in $52 \%$ of the patients. Segmentation software was used to segment osseous 3D models of every hip joint. Validated 3D collision detection software for dynamic impingement simulation using the equidistant method was used.

Results: Most (89-95\%) of the posterior impingement was located extraarticular for both groups and a minority had combined intra- and extraarticular FAI. Posterior impingement occurred between the ischium and the lesser trochanter in all three groups and involved the greater trochanter in $20-40^{\circ}$ of flexion.

Conclusion: Hips with increased FV have complex combined intra- and extraarticular posterior FAI. The patient-specific exact location of impingement can help for planning of hip preservation surgery. The FABER test could be used clinically to detect extraarticular FAI in early flexion $\left(20^{\circ}\right)$. These results can help for planning and to optimize surgical treatment such as hip arthroscopy.

Diagnosing the Anterior Cruciate Ligament's Morphology and Function - Comprehensive Stress MRI of the Human Knee Joint

Sven Nebelung, Eva-Maria Winkelmeyer, Justus Schock, Daniel Truhn. Dusseldorf, Germany; Aachen, Germany

Purpose: To assess the diagnostic value of stress MRI techniques that combine imaging and loading to evaluate staged anterior cruciate ligament (ACL)-deficiency in an arthroscopic ACL transection model.

Materials and Methods: Ten intact human cadaveric knee joints from body donors were subjected to serial MR imaging. Emulating the clinical anterior drawer test, the joint was imaged unloaded $\left(\delta_{0}\right)$ and loaded $\left(\delta_{1}, 147 \mathrm{~N}\right.$ anterior force at the tibia). The imaging protocol comprised clinical standard T1-, T2and Proton Density-weighted sequences with and without fat-saturation and the joint underwent partial and complete arthroscopic ACL transections inbetween imaging. Based on manual segmentations, anatomic landmarks, and central bone axes, the motional changes of the knee were quantified as a function of ACL status and loading by computing 3D Euclidean vectors between i) the femoral and tibial axis-bone-intersections and ii) tibial tuberosity to tip of trochlea and their projections along the $\mathrm{x}-, \mathrm{y}-$, and $\mathrm{z}$-axis. Computed measurements were compared to manual measurements.

Results: To assess the complex loading-induced motional changes, vector magnitudes and their projections along the y-axis were particularly useful to differentiate intact from partial and complete ACL deficiency, e.g. vector projection on y-axis (i): $2.0 \pm 9.1 \mathrm{~mm}$ [intact], $8.1 \pm 8.9 \mathrm{~mm}$ [partial ACL-deficiency], 12.6 $\pm 6.9 \mathrm{~mm}$ [complete], $\mathrm{p}<0.001)$. Manual measurements confirmed these findings.

Conclusion: Beyond providing normative data on tibiofemoral kinematics as a function of ACL status and loading, this study defines a framework to obtain imaging biomarkers of joint stability by simultaneous assessment of ligament structure and function based on stress MRI.
Deep learning for fully-automatic quantification of avascular necrosis of the femoral head on 3D hip MRI in young patients eligible for joint preserving hip surgery: A pilot study

Adrian Ruckli, Till Lerch, Boschung Adam, Simon Steppacher, Nicolas Gerber, Kate Gerber, Jürgen Burger, Moritz Tannast, Klaus Siebenrock, Florian Schmaranzer. Bern, Switzerland

Purpose: Size of necrosis is an important prognostic factor in the management of femoral head necrosis (AVN), usually estimated on radiographs and MRI. Ideally, a fast-volumetric assessment of necrosis size would be desirable. Thus, we evaluated a deep-learning method to automatically quantify the necrotic bone in AVN.

Materials and Methods: IRB-approved retrospective study of 24 patients (mean age 30 years, 9 women) with AVN according to the 2019 ARCO grading: I (negative x-rays): 3 hips; II (no fracture): 4 hips; IIIA (head collapse $<2 \mathrm{~mm}$ ): 10 hips; IIIB (head collapse $>2 \mathrm{~mm}$ ): 7 hips. Patients underwent preoperative 3T hip MRI including $0.8 \mathrm{~mm}^{3}$ 3D T1VIBE on which manual ground truth segmentation of necrosis was performed by an expert reader and then used to train a convolutional neural network (2D U-Net). A 3-fold cross-validation was performed between manual and automatic volumetric analysis of absolute/relative necrosis volume which was compared between early and advanced AVN (ARCO I/II versus IIIA/B).

Results: Mean absolute and relative AVN volume was comparable between manual $\left(8.8 \pm 8 \mathrm{~cm}^{3}, 19 \pm 17 \%\right)$ and automatic $\left(7.2 \pm 6.1 \mathrm{~cm}^{3}, 16\right.$ $\pm 13 \%$ ) segmentation (both $\mathrm{p}>0.05$ ) and showed a strong correlation $\left(r_{p}=0.76\right.$ and 0.74 , both $\left.p<0.001\right)$, respectively. Manual and automated segmentation detected a difference (both $\mathrm{p}<0.05$ ) in relative necrosis volume between early and advanced AVN: $9 \pm 8 \%$ vs $23 \pm 18 \%$ and $9 \pm 7 \%$ vs $18 \pm 14 \%$, respectively.

Conclusion: Applying a deep learning method for volumetric assessment of AVN is feasible and showed good agreement which paves way for evaluation in larger datasets, with the goal to determine it's prognostic value.

Development and Validation of a Multitask Deep Learning Approach to Simultaneously Detect, Segment and Classify Bone Tumors on Radiographs

Claudio E. von Schacky, Nikolas Wilhelm, Valerie Schäfer, Yannik Leonhardt, Sarah C. Foreman, Felix G. Gassert, Florian T. Gassert, Carolin Mogler, Carolin Knebel, Rüdiger von Eisenhart-Rothe, Marcus R. Makowski, Klaus Wörtler, Rainer Burgkart, Alexandra S. Gersing. Munich, Germany

Purpose: To develop and validate a multitask deep learning (DL) model for simultaneous detection, segmentation, and classification of bone tumors on radiographs.

Materials and Methods: This retrospective study analyzed bone tumors observed on 934 radiographs from 934 patients (age 33.2 $\pm 19.3 y, 419$ women) either diagnosed with benign $(n=673)$ or malignant $(n=261)$ bone lesions using histopathology as standard of reference. Overall, 16 tumor entities were included: osteochondromas, enchondromas, chondroblastomas, osteoid osteomas, NOFs, giant cell tumors, chordomas, haemangiomas, simple and aneurysmatic bone cysts, fibrous dysplasia, chondrosarcomas, osteosarcomas, Ewing's sarcomas, plasma cell myelomas, and b cell NHLs. Bounding box placements and segmentations were performed by a radiology resident and reviewed by a fellowship-trained musculoskeletal radiologist. Patients were split $70 \%(\mathrm{n}=654) / 15 \%(\mathrm{n}=140) / 15 \%(\mathrm{n}=140)$ for training, validation, testing. The multitask DL model was based on ResNeXt-101 implemented in PyTorch. The reported model performance was evaluated on the independent test set.

Results: The multitask DL model achieved $83 \%$ accuracy for the classification of bone lesions as malignant or benign. The model achieved an 
accuracy of $51 \%$ for correct subclassification into one of the 16 tumor entities, placed $82 \%$ of the bounding boxes correctly with an intersection over union of $0.52 \pm 34$, and had an average dice score of $0.60 \pm 37$ for segmentations.

Conclusion: The developed multitask DL model allowed for accurate and simultaneous detection, segmentation, and classification of bone tumors on radiographs. Multitask deep learning may improve accuracy by capitalizing on knowledge gained by conducting different tasks simultaneously. An artificial intelligence such as this may assist in diagnostic workflow.

\section{ePoster presentations}

Fast 3D isotropic proton density-weighted fat saturated MRI of the knee at $1.5 \mathrm{~T}$ with compressed sensing: comparison with conventional multiplanar 2D sequences

Christoph H. Endler, Alexander Isaak, Anton Faron, Christoph Katemann, Patrick A. Kupczyk, Dariusch R. Hadizadeh, Ulrike I. Attenberger, Julian A. Luetkens. Bonn, Germany; Hamburg, Germany

Purpose: Compressed sensing (CS) accelerates MRI acquisition by acquiring less data through undersampling of $\mathrm{k}$-space. In this prospective study we evaluated, whether a 3D isotropic proton density-weighted fat saturated sequence (PDwFS) with CS can replace conventional multidirectional $2 \mathrm{D}$ sequences at $1.5 \mathrm{~T}$.

Materials and Methods: 20 patients (45.2 $\pm 20.2 \mathrm{y} ; 10$ women) with suspected internal knee damage received 3D PDwFS with CS acceleration factor 8 (acquisition time: $4: 11 \mathrm{~min}$ ) in addition to standard threeplane 2D PDwFS (acquisition time: 4:05min+3:03min+ $4: 46 \mathrm{~min}=11: 54 \mathrm{~min})$ at $1.5 \mathrm{~T}$. Scores for homogeneity of fat saturation, image sharpness and artifacts were rated by two board-certified radiologists on the basis of 5-point Likert-scales. Based on these ratings, an overall image quality score was generated. Additionally, quantitative contrast ratios for the menisci (MEN), the anterior (ACL) and the posterior cruciate ligament (PCL) in comparison with the popliteus muscle were calculated.

Results: Overall image quality was rated superior in 3D PDwFS compared to $2 \mathrm{D}$ sequences $(14.45 \pm 0.83 \mathrm{vs} .12 .85 \pm 0.99 ; \mathrm{p}<0.01)$, particularly due to less artifacts $(4.65 \pm 0.67 \mathrm{vs} .3 .65 \pm 0.49 ; \mathrm{p}<0.01)$ and a more homogeneous fat saturation $(4.95 \pm 0.22 \mathrm{vs} .4 .55 \pm 0.51 ; \mathrm{p}<0.01)$. Scores for image sharpness were comparable $(4.80 \pm 0.41$ vs. $4.65 \pm 0.49 ; \mathrm{p}=0.30)$. Quantitative contrast ratios for all measured structures were superior in 3D PDwFS (MEN:p<0.05; ACL:p=0.06; PCL:p=0.33). In one case a meniscal tear was only diagnosed using multiplanar reformation of $3 \mathrm{D}$ PDwFS, whereas it would have been missed on standard 2D sequences. Conclusion: An isotropic fat saturated 3D PD-sequence with CS enables fast and high-quality 3D-imaging of the knee at $1.5 \mathrm{~T}$ and may replace conventional multiplanar 2D sequences. Besides faster image acquisition, the $3 \mathrm{D}$ sequence provides advantages in small structure imaging by multiplanar reformation.

Evaluation of MR-derived simulated CT-like images and simulated radiographs of the shoulder joint compared to plain $\mathrm{x}$-rays and $\mathrm{CT}$ images in patients with suspected rotator cuff injury

Georg C. Feuerriegel, Felix Kopp, Daniela Pfeiffer, Yannik Leonhardt, Dimitrios C. Karampinos, Benedikt J. Schwaiger, Marcus R. Makowski, Klaus Woertler, Alexandra S. Gersing. Munich, Germany

Purpose: To evaluate the diagnostic value of MR-derived CT-like images and simulated radiographs compared with conventional radiographs in patients with suspected rotator cuff injury.

Materials and Methods: In 23 patients with suspected rotator cuff injury (mean age 52.2 \pm 18 years,13 women), 3T MR-imaging of the shoulder was performed including a 3D T1-weighted gradient-echo sequence from which intensity-inverted CT-like images were derived. The inverted MRI-image volumes were processed to achieve contrast enhancement and a cone-beam forward projection algorithm was used to obtain simulated radiographs. Congruency of projections and image quality was graded using a 5-point Likert-scale. Two radiologists evaluated the simulated images and corresponding conventional radiographs, performing measurements of the acromiohumeral distance(AHD) and critical shoulder angle(CSA), as well as assessing semi-quantitative scores in order to grade degenerative changes using the Samilson-classification and the type of the acromion shape (Bigliani-classification).

Results: Measurements on simulated radiographs were comparable to those on conventional radiographs with a substantial to almost perfect inter- and intrareader agreement $(K=0.69-1.00$ and $K=0.65-0.85$, respectively). Between MR-derived CT-like images in combination with simulated radiographs and conventional radiographs a substantial agreement was found regarding diagnostically relevant features, assessed on $\mathrm{Y}$-and AP-projections (CSA, mean+/- standard deviation $33^{\circ}+/-3.3^{\circ}, \mathrm{K}=0.84$ and 0.69; AHD, mean+/-SD, $11.8 \mathrm{~mm}+/-1.9 \mathrm{~mm}, \mathrm{k}=0.95$ and 0.60 ; Samilson-score, $\mathrm{K}=0.77$ and 0.77 ; Bigliani-score, $\mathrm{k}=0.83$ and 0.67 , respectively).

Conclusion: The visualization and assessment of diagnostically relevant features of shoulder joints was feasible using MRderived CT-like images and simulated radiographs and findings were comparable to those of conventional radiographs. CT-like images may provide additional information on osseous abnormalities without causing any radiation exposure.

Magnetic resonance observation of cartilage repair tissue (MOCART) for the evaluation of retropatellar autologous chondrocyte transplantation and correlation to clinical outcome: Surface of repair tissue and subchondral bone changes should be the focus of attention

Sophia S.Goller, Andreas Heuck, Bernd Erber, Nicola Fink, Thomas R. Niethammer, Peter E. Müller, Jens Ricke, Andrea Baur-Melnyk. Munich, Germany

Purpose: Matrix-associated autologous chondrocyte transplantation (MACT) has become an established method for the treatment of cartilage defects. Therefore, the purpose of this study was to systematically evaluate retropatellar cartilage grafts with regard to distinct MR-morphological criteria and correlation to clinical outcome.

Materials and Methods: 38 patients with a mean age of 31,6 years and having received MACT of mostly one singular retropatellar cartilage defect $(95.0 \%)$ were included in this study. Postoperative MRI was performed after 3, 6 and 12 months and systematically evaluated using a modified Magnetic Resonance Observation of Cartilage Repair Tissue (MOCART) scoring system. The clinical status of the patients was determined preoperatively and 12 months postoperatively using International Knee Documentation Committee Subjective Form (IKDC), which was correlated with the MRI data.

Results: After 12 months an overall clinical improvement as measured by IKDC values was observed $(39.9 \pm 19.1$ vs. $57.5 \pm 21.4$; $\mathrm{p}=0.0003)$. There was a statistically significant association between alterations in the surface of the repair tissue $(p=0.0068)$ as well as changes in the subchondral bone $(\mathrm{p}=0.0329)$ compared with the clinical outcome after 12 months. In contrast, all other MRI variables did not show significant association to clinical outcome at the end of the observation period.

Conclusion: In postoperative MRI follow-up after MACT treatment for retropatellar cartilage defects radiologists should draw special attention both to the surface of the repair tissue as well as to changes in subchondral bone, as they are associated with clinical outcome 12 months postoperatively. 
All trauma or what? Sonography of the elbow joint on the growing skeleton

\section{Christina Hauenstein, Malte Jäschke. Rostock, Germany}

Purpose: Injuries to the child's elbow are common, but not every trauma results in a fracture. Knowledge of the differential diagnoses including inflammatory and tumorous processes in pain and limited movement of the elbow is important to make the correct diagnosis. Case studies will be used to demonstrate the possibilities of sonography of the elbow joint in paediatrics and paediatric traumatology.

Materials and Methods: Processing of case studies from the last 2 years from the paediatric radiology department of the University Medical Center Rostock.

Results: The sonography of the elbow joint allows to set the course for further diagnostics and therapy of the patients without much effort. This is particularly important since it is not always possible to establish a reliable trauma history in children and the clinical picture of pain and the resulting relieving posture, which is usually leading, requires further diagnostic classification. An advantage of sonography are functional examinations in pro- and supination as well as in bent and stretched elbow joints. A side comparison is obligatory and helps to identify osseous lesions and soft tissue involvement. The detection of an effusion or haemarthros in the sense of a sonographic "fad pat sign" has a very high diagnostic predictive value.

Conclusion: Sonography is an excellent method, especially in younger children, to locate pathologies of the elbow joint and with sufficient experience to interpret them correctly.

\section{Natural language processing enables analysis of fracture frequency} before and during the COVID-19 pandemic

Tobias Jorg, Felix Hahn, Philipp Mildenberger, Christoph Düber, Daniel Wagner, Roman Kloeckner, Florian Jungmann. Mainz, Germany

Purpose: People's daily routines changed considerably during the rise of COVID-19 in Germany. While there was a rising number of chest-CTs and x-rays for diagnosing COVID-19 radiology was also confronted with indirect effects as in decreasing numbers of patients in need of trauma-related imaging being present in the emergency department.

In this study Natural language processing (NLP) was used to analyze total numbers, and distribution of fractures found in radiographs during the COVID-19 pandemic, and in the five years before.

Materials and Methods: We used NLP to automatically categorize 5397 radiological reports of radiographs (hand/wrist, elbow, shoulder, ankle, knee, pelvis/hip) within a six-week period from March to April for the years 2015 to 2020 into "fracture" or "no fracture". We trained an existing NLP-engine to detect fractures and reached an F1-score of 0.79 .

Results: In 2020, we found a significant decrease of fractures in general $(p<0.001)$. The average number of fractures of the years 2015-2019 was 295 , whereas it was only 233 in 2020 . In children and adolescents $(\mathrm{p}<0.001)$, and in adults $(\mathrm{p}=0.006)$, significantly less fractures were reported in 2020. The number of fractures among the elderly ( $>65$ years) did not change $(\mathrm{p}=0.15)$.

The number of hand/wrist fractures $(\mathrm{p}<0.001)$ and fractures of the elbow $(p<0.001)$ was significantly lower in 2020 compared to the years 2015 2019.

Conclusion: NLP is capable of identifying changes in the number of pathologies as shown here for the use case fracture detection. This may trigger root cause analysis and enable automated real time monitoring in radiology.
The predictive Value of early postoperative subchondral Bone Marrow Parameters for mid-Term Outcome after MACI with autologous Bone Grafting at the knee: A quantitative longitudinal 3T MRI Study

Matthias Jung, Stefan Ruschke, Dimitrios C. Karampinos, Christian Holwein, Thomas Baum, Alexandra S. Gersing, Andreas B. Imhoff, Fabian Bamberg, Pia M. Jungmann. Freiburg, Germany; Munich, Germany

Purpose: To longitudinally assess, whether early postoperative quantitative 3T MRI parameters of subchondral bone marrow are able to predict the 2-year clinical and MRI outcome after matrix-associated autologous chondrocyte implantation (MACI) with autologous bone grafting (ABG) at the knee.

Materials and Methods: A total of $\mathrm{N}=18$ patients who received MACI with ABG for treatment of focal osteochondral defects at the medial $(n=15)$ or lateral $(n=3)$ femoral condyle at the knee received MRI follow-up (FU) 3, 6, 12 and 24 months postoperatively. Quantitative MRI included bone marrow edema (BME) volume measurements and single-voxel MR spectroscopy (MRS; $n=9$ ) of the subchondral bone marrow. At 2-year follow-up, morphological MRI outcome included MOCART 2.0 scores. Clinical outcomes were assessed using Lysholm scores.

Results: Mean BME volume showed a decrease from $4.9 \mathrm{~cm}^{3}$ at 3 months to $2.0 \mathrm{~cm}^{3}$ at 2-year $\mathrm{FU}(\mathrm{P}=0.033)$. MRST2 showed a significant decrease from $20.7 \mathrm{~ms}$ at 1 -year $\mathrm{FU}$ to $16.8 \mathrm{~ms}$ at 2 -year FU ( $\mathrm{P}=0.040)$. The difference was not significant for the MRS PD water fraction (PDWF; $\mathrm{P}=0.247)$ and the unsaturated lipid fractions $(\mathrm{P}=0.347)$. Higher $\mathrm{BME}$ volume at 6 months correlated significantly with worse 2-year clinical outcomes (Lysholm scores; $\mathrm{R}=-0.616 ; \mathrm{P}=0.015$ ) and 2-year MOCART scores $(\mathrm{R}=-0.567 ; \mathrm{P}=0.027)$. Correlations for PDWF, MRST2 and unsaturated lipid fractions were not significant $(\mathrm{P}>0.05)$.

Conclusion: Quantitative subchondral bone parameters improved over time. Low subchondral BME volume at early postoperative timepoints predict better 2-year clinical and MRI outcomes after MACI with ABG.

Early diagnosis of osteonecrosis in children with ALL and LBL during antileukemic therapy using diffusion and perfusion in MRI results from the OPAL study (Osteonecrosis in Pediatric Patients with ALL and LBL)

Dirk Klee, Hans-Joerg Wittsack, Hans-Juergen Laws, Gerald Antoch, Joerg Schaper, Katharina Weil. Dusseldorf, Germany

Purpose: In children with acute lymphoblastic leukemia (ALL) and lymphoblastic lymphoma (LBL), aseptic osteonecrosis $(\mathrm{ON})$ is a common side effect of therapy, which affects the quality of life of the patients. The OPAL study evaluates the value of MRI for the early diagnosis of ON, using diffusion and perfusion percentages in ADC maps to make a prospective statement on the occurrence of $\mathrm{ON}$.

Materials and Methods: In 19 patients, ADC measurements of diffusion and perfusion proportions from a bi-exponential DWI analysis were performed in the dia-, meta- and epiphysis of the distal femur and proximal tibia at the time of diagnosis, after 6, 9 and 12 months. Further, anatomical images using STIR and T1-weighted MRI were recorded.

Results: The leukemic infiltrations initially represented hyper- and hypointensity in the STIR and T1. After 6 and partly 9 months in most patients an inverse signal behavior compared to the initial examination and a decrease of signal intensity over time analogous to STIR were detected. Due to this diffusion behavior, no valid results could be obtained with the bi-exponential IVIM ("intravoxel incoherent motion") model for analysis of the DWI data on the diaphysis. At the time of 12 months, ON with a hyperintense signal (STIR and DWI) distinguished from the environment. No significant differences in the preliminary investigations 
between regions with later $\mathrm{ON}$ and without $\mathrm{ON}$ could be detected in diffusion and perfusion.

Conclusion: A prospective statement on the development of $\mathrm{ON}$ in under antileukemic therapy is not successful by diffusion and perfusion in MRI with the used methods.

\section{Biomechanics meets Imaging - Effects of Loading on Cartilage Tissue} Functionality

Sven Nebelung, Ken Tonio Zwingenberger, Justus Schock, Daniel Truhn. Dusseldorf, Germany; Aachen, Germany

Purpose: Biomechanical MRI of articular cartilage, i.e. its imaging under loading, is promising to assess the tissue's functionality in health and disease. Using serial T1 $\rho$ mapping as an exquisitely mechanosensitive quantitative MRI parameter, this study assessed the responses to static and dynamic loading of histologically intact cartilage samples under pressure-controlled in-situ loading.

Materials and Methods: 47 cartilage samples were obtained from the medial femoral condyles of total knee arthroplasties, prepared to standard thickness, and placed in a standard knee joint in a pressure-controlled whole knee-joint compressive loading device. Cartilage samples' responses to static (i.e. constant), dynamic (i.e. alternating), and no loading were assessed before $\left(\delta_{0}\right)$, and after $30 \mathrm{~min}\left(\delta_{1}\right)$ and $60 \mathrm{~min}\left(\delta_{2}\right)$ of loading using serial T1 $\rho$ maps acquired on a 3.0T clinical MRI scanner (Achieva, Philips). Relative changes in $\operatorname{T} 1 \rho\left(\Delta_{1}, \Delta_{2}\right)$ were determined for the upper and lower sample halves and the entire sample, analysed using appropriate statistical tests, and referenced to histologic (Mankin scoring) and biomechanical reference measures (tangent stiffness).

Results: Histologic, biomechanical, and T1 $\rho$ sample characteristics at $\delta_{0}$ were relatively homogenous in all samples. In response to loading, relative increases in T1 $\rho$ were significant after dynamic loading $\left(\Delta_{1}=10.3\right.$ $\pm 17.0 \%, \Delta_{2}=21.6 \pm 21.8 \%, \mathrm{p}=0.002$ ), while relative increases in T1 $\rho$ after static loading and in controls were only moderate and not significant. Conclusion: When realizing the clinical translation, this in-situ study suggests that serial T1 $\rho$ mapping is best combined with dynamic loading to assess cartilage functionality in humans based on advanced MRI techniques.

Publisher's note Springer Nature remains neutral with regard to jurisdictional claims in published maps and institutional affiliations. 